K. Watanabe

Nagoya Math. J.

Vol. 49 (1973), 101-109

\title{
SOME EXAMPLES OF ONE DIMENSIONAL GORENSTEIN DOMAINS
}

\author{
KEIICHI WATANABE
}

\section{Introduction.}

In this paper, I will prove the following theorems;

THEOREM 1. For given integers $n$ and $m$ such that $m \geqq 2^{n}$, there exist 1-dimensional local domains which are complete intersections and have embedding dimension $n+1$ and multiplicity $m$.

THEOREM 2. For given integers $n$ and $m$ such that $4 \leqq n \leqq m-1$, there exist 1-dimensional local domains which are Gorenstein with multiplicity $m$ and embedding dimension $n$ and which are not complete intersections.

To give these examples I heavily use the theory of the value-semigroups of 1-dimensional local domains by Kunz and Herzog ([1], [3]).

$\S 1$. Review of the theory of value-semigroups of 1-dimensional local domains ([1], [3]).

In the following, a 'semigroup' always means an additive subsemigroup of $N$, the additive semigroup of non-negative integers.

(1) A 'numerical semigroup' is a semigroup $H$ which satisfies two conditions ;

1. $0 \in H$

2. There exists an integer $c$ such that any integer $n \geqq c$ is in $H$.

(2) The conductor of a numerical semigroup $H$, denoted by $c(H)$, is the smallest integer $c$ such that all integers $n$ satisfying $n \geqq c$ belong to $H$.

(3) We denote by $\left\langle n_{1}, \cdots, n_{k}\right\rangle$ the semigroup generated by $n_{1}, \cdots$, $n_{k} ;\left\langle n_{1}, \cdots, n_{k}\right\rangle=\left\{\sum_{i=1}^{k} a_{i} n_{i} \mid a_{i} \in N\right\}$.

Received December 7, 1971.

Revised August 1, 1972. 
(4) We say that $\left\{n_{1}, \cdots, n_{k}\right\}$ is the minimal generator system of a semigroup $H$ if $H=\left\langle n_{1}, \cdots, n_{k}\right\rangle$ and any proper subset of $\left\{n_{1}, \cdots, n_{k}\right\}$ does not generate $H$. If we suppose that $n_{1}<n_{2}<\cdots<n_{k}$, this is equivalent to say that $n_{i} \notin\left\langle n_{1}, \cdots, n_{i-1}\right\rangle$ for $2 \leqq i \leqq k$.

When we write $H=\left\langle n_{1}, \cdots, n_{k}\right\rangle$, we agree that $\left\{n_{1}, \cdots, n_{k}\right\}$ is the minimal generator system. Minimal generator system of an arbitrary subsemigroup of $N$ uniquely exists.

(5) A numerical semigroup $H$ is symmetric if for any integer $n$, $n \in H \Leftrightarrow c-1-n \notin H(c=c(H))$.

(6) $K[H]=K\left[T^{h} ; h \in H\right] \subset K[T]$ ( $K$ is a field and $T$ is an indeterminant). $K[H]_{\text {loc }}$ the localization of $K[H]$ 'at the origin'. If $H$ is a numerical semigroup, the integral closure of $K[H]$ in the quotient field of $K[H]$ is $K[T]$.

If $H=\left\langle n_{1}, \cdots, n_{k}\right\rangle$, then $K[H]=K\left[T^{n_{1}}, \cdots, T^{n_{k}}\right]$.

(7) We say that a numerical semigroup $H$ is a complete intersection if the ring $K[H]$ is a complete intersection. When $H=\left\langle n_{1}, \cdots, n_{k}\right\rangle$, and if we consider the homomorphism $\Phi_{H}: K\left[X_{1}, \cdots, X_{k}\right] \rightarrow K[H]$, $\Phi_{H}\left(X_{i}\right)=T^{n_{i}}, H$ is a complete intersection if and only if $\operatorname{Ker}\left(\Phi_{H}\right)$ is generated by $k-1$ elements.

(8) The multiplicity of $H$, denoted by $m(H)$ is the least positive integer in $H$. If $H=\left\langle n_{1}, \cdots, n_{k}\right\rangle$ with $n_{1}<n_{2}<\cdots<n_{k}$, then $m(H)=n_{1}$.

(9) The embedding dimension of $H$, denoted by emb $(H)$, is the number of the minimal generators of $H$. If $H=\left\langle n_{1}, \cdots, n_{k}\right\rangle$, then $\operatorname{emb}(H)=k$ (recall that $\left\{n_{1}, \cdots, n_{k}\right\}$ is the minimal generator system).

(10) Let $H=\left\langle n_{1}, \cdots, n_{k}\right\rangle$ and $h \in H$. If $h$ has different expressions as linear combinations of $n_{i}$ 's, then we say that $h$ is a relation of $H$. For example, if $H=\langle 3,4,5\rangle, 8=2 \cdot 4=3+5$ and $9=3 \cdot 3=4+5$ are relations of $H$.

(11) For a relation $h$ in $H=\left\langle n_{1}, \cdots, n_{k}\right\rangle$, we associate to $h$ a vector $v_{h} \in Z^{k}$ in the following way. If $h=\sum_{i=1}^{k} a_{i} n_{i}=\sum_{i=1}^{k} b_{i} n_{i}$, then $v_{h}=\left(a_{1}-b_{1}, a_{2}-b_{2}, \cdots, a_{k}-b_{k}\right)$. In the example in (10), $v_{8}=(-1,2$, $-1)$, and $v_{9}=(3,-1,-1)$. Of course $v_{h}$ is not determined uniquely by $v_{h}$. But as it is not important in our following arguments, we agree to fix one such $v_{h}$.

(12) For $H=\left\langle n_{1}, \cdots, n_{k}\right\rangle$, we define; 


$$
M(H)=\min \left\{\begin{array}{l|l}
h_{1}+h_{2}+\cdots+h_{k-1} & \begin{array}{l}
h_{1}, \cdots, h_{k-1} \text { are relations in } H \text { and } \\
v_{h_{1}}, \cdots, v_{h_{k-1}} \text { are linearly } \\
\text { independent in } \boldsymbol{Z}^{k}
\end{array}
\end{array}\right\}
$$

For example, if $H=\langle 3,4,5\rangle, M(H)=8+9=17$.

Let $R$ be an analytically irreducible 1-dimensional Noetherian local domain. Then the integral closure $V$ of $R$ in the quotient field of $R$ is a discrete valuation ring. We assume that $R$ and $V$ has the same residue class field. (Which is true if $R=K[H]_{\text {loc }}$ ) If we denote by $v$ the valuation attached to $V$, then $H_{R}=v(R)$ is a numerical semigroup and we have the following propositions.

Proposition 1. (1) Multiplicity of $R=m\left(H_{R}\right)$.

(2) Embedding dimension of $K[H]_{10 \mathrm{c}}=\mathrm{emb}(H)$,

(3) $R$ is Gorenstein if and only if $H_{R}$ is symmetric.

(4) If $H_{R}$ is a complete intersection, then $R$ is a complete intersection.

(4') If $R=K[H]_{1 \mathrm{oc}}$, then the converse of (4) holds.

Proposition 2. ([1], Satz 5.10) If $H=\left\langle n_{1}, \cdots, n_{k}\right\rangle$, then we have that $M(H)-\sum_{i=1}^{k} n_{i}+1 \geqq c(H)$, and the equality holds if and only if $H$ is a complete intersection.

§ 2. Examples of 1-dimensional local domains which are complete intersections and have given embedding dimension and multiplicity.

LEMMA 1. Let $H_{1}=\left\langle n_{1}, \cdots, n_{k}\right\rangle, a$ and $b$ be positive integers such that;

(i) $a \in H_{1}$ and $a \neq n_{i}(i=1, \cdots, k)$.

(ii) $a$ and $b$ are relatively prime.

Then, if we put $H=\left\langle a, b n_{1}, \cdots, b n_{k}\right\rangle$ (which we will denote by $H=$ $\left.\left\langle a, b H_{1}\right\rangle\right)$, we have;

(1) $H$ is a complete intersection if and only if $H_{1}$ is a complete intersection.

(2) $H$ is symmetric if and only if $H_{1}$ is symmetric.

Proof. We consider the canonical homomorphisms $\Phi_{1}: K\left[Y_{1}, \cdots, Y_{k}\right]$ $\rightarrow K\left[H_{1}\right]$ and $\Phi: K\left[Y_{1}, \cdots, Y_{k}, X\right] \rightarrow K[H]$ defined by $\Phi_{1}\left(Y_{i}\right)=T^{n_{i}}, \Phi(X)=$ $T^{a}, \Phi\left(Y_{i}\right)=T^{b n_{i}}(i=1,2, \cdots, k)$. We put $\operatorname{Ker}\left(\Phi_{1}\right)=A_{1}$ and $A=\left(A_{1}, X^{b}\right.$ $\left.-Y_{1}^{e_{1}} Y_{2}^{e_{2}} \cdots Y_{k}^{e_{k}}\right)$ where $e_{1}, \cdots, e_{k}$ are defined by $a=\sum_{i=1}^{k} e_{i} n_{i}$ (we fix one 
such expression). We claim $\operatorname{Ker}(\Phi)=A$. $\operatorname{Ker}(\Phi) \supset A$ is obvious. Conversely, if $f\left(X, Y_{1}, \cdots, Y_{k}\right) \in \operatorname{Ker}(\Phi)$, we can find $f_{0}(Y), \cdots, f_{b-1}(Y) \in$ $K\left[Y_{1}, \cdots, Y_{k}\right]$ such that $f \equiv f_{0}+X f_{1}+\cdots+X^{b-1} f_{b-1}(\bmod A)$. Hence $\Phi\left(f_{i}\right) \in K\left[T^{b}\right]$. As $\Phi\left(X^{i}\right)=T^{a i}$ and $(a, b)=1$, it follows from $\Phi(f)=0$, that $\Phi\left(f_{i}\right)=0$, i.e. $f_{i} \in \operatorname{Ker}\left(\Phi_{1}\right)=A_{1}(i=1, \cdots, b-1)$.

Now, we have $K[H]=K\left[H_{1}\right][X] /(g)$, where $g(X, Y)=X^{b}-Y_{1}^{e_{1}} \cdots Y_{k}^{e_{k}}$. Thus we have; $K[H]$ is a complete intersection (resp. Gorenstein) $\Leftrightarrow K\left[H_{1}\right][X]$ is a complete intersection (resp. Gorenstein) $\Leftrightarrow K\left[H_{1}\right]$ is a complete intersection (resp. Gorenstein). By Proposition 1 we are done.

By Lemma 1, we have semigroups which are complete intersections and have arbitrarily high embedding dimensions. When embedding dimension is $\leqq 3$, the converse holds.

Proposition 3. If $H$ is a semigroup which is a complete intersection and if $e m b(H)=3$, then $H=\left\langle a, b H_{1}\right\rangle$ where $H_{1}$ is a semigroup of $e m b\left(H_{1}\right)=2$ (which is necessarily a complete intersection) and $a$ and $b$ are integers satisfying the conditions of Lemma 1.

(This proposition is proved by Herzog [2]. But as his proof is considerably long, I give a shorter proof.)

To prove the proposition, we need a lemma.

LEMMA 2. Let $H=\left\langle n_{1}, \cdots, n_{k}\right\rangle$ be a semigroup which is a complete intersection, $\Phi_{H}: K\left[X_{1}, \cdots, X_{k}\right] \rightarrow K[H]$ be the canonical homomorphism and $\left(f_{1}, \cdots, f_{k-1}\right)$ the generators of $\operatorname{Ker}\left(\Phi_{H}\right)$. If we denote by $J_{p}$ the ideal generated by $p$ variables $X_{i_{1}}, \cdots, X_{i_{p}}$, then there exists at most $p-1 f_{i}^{\prime}$ 's which belong to $J_{p}$.

COROLlary. For every variable $X_{j}(1 \leqq j \leqq k)$, one of the $f_{i}$ 's includes a monomial of the type $X_{j}^{s}$.

Proof of Lemma 2. We consider the ideal $A=\left(J_{p}, f_{1}, \cdots, f_{k-1}\right)$. If $f_{1}, \cdots, f_{p} \in J_{p}$ (for simplicity, we renumber $f_{i}^{\prime}$ 's), then $A=\left(J_{p}, f_{p+1}, \cdots, f_{k-1}\right)$ and $A$ is generated by $k-1$ elements and $h t(A)$ must be $\leqq k-1$. But on the other hand, $\operatorname{dim}(K[H])=1$ and $\Phi_{H}\left(J_{p}\right) \neq 0$. So, we must have $h t(A)=k$. Contradiction !

The corollary is a special case of the lemma when $p=k-1$.

Proof of Proposition 3. Let $H=\left\langle n_{1}, n_{2}, n_{3}\right\rangle, \Phi_{H}: K\left[X_{1}, X_{2}, X_{3}\right] \rightarrow K[H]$, $\operatorname{Ker}\left(\Phi_{H}\right)=\left(f_{1}, f_{2}\right)$. By the definition of $\Phi_{H}$, each $f_{i}$ is of the form 
(monomial)-(monomial). Then, by the corollary of Lemma 2, after renumbering $X_{i}$ 's and $f_{i}^{\prime}$ 's, we may assume, $f_{1}=X_{2}^{n}-X_{3}^{m}, f_{2}=X_{1}^{b}-$ $X_{2}^{e} X_{3}^{f}$. As $\left(f_{1}, f_{2}\right)$ is a prime ideal of height $2, f_{1}$ and $f_{2}$ must be irreducible and we have $(m, n)=1, n \cdot n_{2}=m \cdot n_{3}, b n_{1}=e n_{2}+f n_{3}$. We put $H_{1}=\langle m, n\rangle, n_{1}=a, n_{2}=d m, n_{3}=d n$. Then $a b=d(e m+f n)$. From $\left(n_{1}, n_{2}, n_{3}\right)=1$, we have $(a, d)=1$ and $b=d b^{\prime}$. We claim that $d=b$ and $a=e m+f n$. Let us assume $d \neq b, b^{\prime} \neq 1$. If $a \notin H_{1}$, take an integer $s$ such that $s a \in H_{1}$ and $s$ is not a multiple of $b^{\prime}$. Then, writing $s a=$ $e^{\prime} m+f^{\prime} n, g=X_{1}^{s d}-X_{2}^{e^{\prime}} X_{3}^{f^{\prime}} \in \operatorname{Ker}\left(\Phi_{H}\right)$. But it is easy to assure that $g \oplus\left(f_{1}, f_{2}\right)$. This contradicts the fact that $\left(f_{1}, f_{2}\right)=\operatorname{Ker}\left(\Phi_{H}\right)$. If $a \in H_{1}$, $a=e^{\prime} m+f^{\prime} n$, then $X_{1}^{d}-X_{2}^{e^{\prime}} X_{3}^{f^{\prime}} \in \operatorname{Ker}\left(\Phi_{H}\right)$. From $\operatorname{Ker}\left(\Phi_{H}\right)=\left(f_{1}, f_{2}\right)$, we get $d=b$.

Remark 1. Proposition 3 is not true if $\operatorname{emb}(H) \geqq 4$. For example, If we put $H=\langle 14,21,15,20\rangle, H$ is a complete intersection with $c(H)=$ $68, \operatorname{Ker}\left(\Phi_{H}\right)=\left(X_{1}^{3}-X_{2}^{2}, X_{1} X_{2}-X_{3} X_{4}, X_{3}^{4}-X_{4}^{3}\right)$ and clearly $H$ can not be written in the form $H=\left\langle a, b H_{1}\right\rangle$.

Remark 2. By Proposition 3, we can determine the types of $H^{\prime}$ s which are complete intersections and $e m b(H) \leqq 3$. For example, if $e m b(H)=3$ and $m(H)=5$ and if $H$ is a complete intersection, (this is equivalent to say that $H$ is symmetric, in this case) then $H=\langle 5,2 p, 3 p\rangle$, $p \geqq 3,(p, 5)=1$.

LEMMA 3. Let a be an odd integer. Then the semigroup $H=$ $\left\langle 2^{n}, 2^{n}+a, 2^{n}+2 a, \cdots, 2^{n}+2^{i} a, \cdots, 2^{n}+2^{n-1} a\right\rangle$ is a complete intersection for $n \geqq 1$.

Proof. Easy by induction and applying Lemma 1.

THEOREM 1. Let $m$ and $n$ be given positive integers such that $m \geqq 2^{n}$. Then there exists a 1-dimensional local domain $R$ which is a complete intersection with embedding dimension $n+1$ and multiplicity $m$.

Proof. We find a semigroup $H$ which is a complete intersection and $m(H)=m, e m b(H)=n+1$.

(i) If $m$ is odd, we put $m=2^{n}+a$. Then, by Lemma $3, H_{1}=$ $\left\langle 2^{n-1}, 2^{n-1}+a, \cdots, 2^{n-1}+2^{n-2} a\right\rangle$ is a complete intersection and $m \in H_{1}$. If we take an integer $b$, such that $(b, m)=1$ and $2^{n-1} b \geqq m$, then $H=$ $\left\langle m, b H_{1}\right\rangle$ is the desired semigroup. 
(ii) If $m$ is even, using induction on $n$, we may assume that there exists a semigroup $H_{1}$ which is a complete intersection and $m\left(H_{1}\right)=m / 2$, $e m b\left(H_{1}\right)=n$. Then, if we take an odd integer $a \in H_{1}$ such that $a>m$ and $a$ is not a generator of $H_{1}, H=\left\langle a, 2 H_{1}\right\rangle$ is the desired semigroup by Lemma 1 .

Remark. If $(R, M)$ is a regular local ring and if $\left(x_{1}, \cdots, x_{n}\right)$ is a regular sequence of $\mathrm{R}$ contained in $M^{2}$, then the multiplicity of $R /\left(x_{1}, \cdots, x_{n}\right)$ is at least $2^{n}$. So the condition $m \geqq 2^{n}$ is necessary.

§3. Examples of 1-dimensional Gorenstein local domains which are not complete intersections.

LEMMA 4. Let $m$ and $n$ be positive integers such that $m-1 \geqq n$ $\geqq 4$. If there exist integers $a, b, e$ such that

(i) $a, b \geqq 0$ and $e>0$,

(ii) if $b>0$, then $e$ is even,

(iii) $e a+(e / 2) b+2=m$ (if $e$ is odd, then $b=0$ ),

(iv) $n=a+b+1$.

Then there exists a symmetric semigroup $H$ with $m(H)=m$ and $\operatorname{emb}(H)$ $=n$ and $H$ is not a complete intersection. Actually,

$$
H=\langle m, m+1, \cdots, m+a, 2 m-b, 2 m-b+1, \cdots, 2 m-1\rangle .
$$

Proof. We have $c(H)=e(m+a)+2$. It is easy to see that $H$ is symmetric. To prove that $H$ is not a complete intersection, we restrict ourselves to the case $a>0$. (The case $a=0$ can be proved similarly. But as the case $a=0$ is not used later, we omit the proof.) We give two different proofs, the first one using Proposition 2 and the second one using Lemma 2,

First proof. We compute $M(H)$. In the notation of $\S 1$, (12), we have ;

$$
\begin{aligned}
& h_{1}=2 m+2=m+(m+2)=2(m+1) \\
& h_{2}=2 m+3=m+(m+3)=(m+1)+(m+2) \\
& \ldots \ldots \ldots \ldots \ldots \ldots \ldots \ldots \ldots \ldots \ldots \\
& h_{a-1}=2 m+a=m+(m+a)=(m+1)+(m+a-1) \\
& h_{a}=3 m-b+1=m+(2 m-b+1)=(m+1)+(2 m-b) \\
& h_{a+1}=3 m-b+2=m+(2 m-b+2)=(m+1)+(2 m-b+1)
\end{aligned}
$$




$$
\begin{aligned}
& h_{a+b-1}=3 m=(m+1)+(2 m-1) \\
& h_{a+b}=c(H)+m-b=(e / 2+1)(2 m-b) .
\end{aligned}
$$

$M(H)-\sum_{i=1}^{n} n_{i}+1-c(H)=m b+m(a-2)=(n-3) m>0$. By Proposition $2, H$ is not a complete intersection.

Second proof. We consider the canonical homomorphism $\Phi_{H}: K\left[X_{0}\right.$, $\left.X_{1}, \cdots, X_{a}, X_{a+1}, \cdots, X_{a+b}\right] \rightarrow K[H]$, defined by $\Phi_{H}\left(X_{i}\right)=T^{m+i}(0 \leqq i \leqq a)$, $\Phi_{H}\left(X_{j}\right)=T^{2 m-a-b-1+j}(a+1 \leqq j \leqq a+b)$. We assume that $\operatorname{Ker}\left(\Phi_{H}\right)$ is generated by $a+b$ elements and lead to a contradiction. By the definition of $\Phi_{H}$, it is clear that $f_{1}=X_{0} X_{2}-X_{1}^{2}, f_{2}=X_{0} X_{3}-X_{1} X_{2}, \cdots, f_{a-1}=$ $X_{0} X_{a}-X_{1} X_{a-1}, f_{a}=X_{0} X_{a+2}-X_{1} X_{a+1}, \cdots, f_{a+b-1}=X_{1} X_{a+b}-X_{0}^{3}$ are $a+b$ -1 members of minimal generators of $\operatorname{Ker}\left(\Phi_{H}\right)$. As $\operatorname{Ker}\left(\Phi_{H}\right)$ is generated by $f_{1}, \cdots, f_{a+b-1}$ and one more polynomial $g$, and as $g$ can include at most 2 monomials of the form $X_{i}^{s}$, we have $a+b+1 \leqq 4$. It remains to show that $a+b+1=4$ does not occur. If $a=1, b=2$, then $f_{1}=$ $X_{0} X_{3}-X_{1} X_{2}$ and $f_{2}=X_{1} X_{3}-X_{0}^{3}$. So it is impossible to find $f_{3}$ satisfying the condition of the corollary of Lemma 2. If $a=2, b=1$, then $f_{1}=$ $X_{0} X_{2}-X_{1}^{2}$ and $f_{2}=X_{1} X_{3}-X_{0}^{3}$. But in this case, $f_{1}, f_{2} \in\left(X_{0}, X_{1}\right)$ and this contradicts Lemma $2(p=2)$. If $a=3, b=0, f_{1}=X_{0} X_{2}-X_{1}^{2}$ and $f_{2}=$ $X_{0} X_{3}-X_{1} X_{2}$ and it is impossible to find $f_{3}$ satisfying the condition of the corollary of Lemma 2. This concludes the proof of Lemma 4.

LEMMA 5. If $m-1 \geqq n \geqq m / 2$, there exist $a, b$ and $e$ satisfying the conditions of Lemma 4. Furthermore, we can take $a>0$.

Proof. Put $e=2, b=2 n-m, a=m-n-1$ if $n \neq m-1$. When $n=m-1$, we put $e=1, b=0, a=n-1=m-2$.

LEMMA 6. If $m \geqq 5$, there exists a symmetric semigroup $H$, which is not a complete intersection and with $m(H)=m$, emb $(H)=4$.

Proof. Case I $m \equiv 1(\bmod 4)$. Writing $m=4 m^{\prime}+1$, we put

$$
H=\left\langle m, m+1, m+2, m^{\prime}(m+2)+1\right\rangle \text {. }
$$

Then $H$ is symmetric with;

$$
c(H)=2 m^{\prime} m, \quad M(H)=h_{1}+h_{2}+h_{3},
$$

where

$$
h_{1}=2 m+2=2(m+1)=m+(m+2)
$$




$$
\begin{aligned}
& h_{2}=\left(m^{\prime}(m+2)+1\right)+m=m^{\prime}(m+2)+(m+1) \\
& h_{3}=c(H)+m=\left(2 m^{\prime}+1\right) m=\left(m^{\prime}(m+2)+1\right)+m^{\prime}(m+2) .
\end{aligned}
$$

$M(H)-m-(m+1)-(m+2)-\left(m^{\prime}(m+2)+1\right)+1-c(H)=m>0$, $H$ is not a complete intersection.

Case II $m \equiv 2(\bmod 4)$. In the Lemma 4 , put $a=1, b=2, e=$ $(m-2) / 2$.

Case III $m \equiv 3(\bmod 4)$. We put

$$
H=\langle m, m+1,2 m+3,2 m+4\rangle \text {. }
$$

Then $H$ is symmetric with;

$$
c(H)=\frac{m(m+1)}{2}, \quad M(H)=h_{1}+h_{2}+h_{3},
$$

where

$$
\begin{aligned}
& h_{1}=3 m+3=3(m+1)=m+(2 m+3) \\
& h_{2}=3 m+4=(m+1)+(2 m+3)=m+(2 m+4) \\
& h_{3}=c(H)+m=(2 m+3)+\frac{m-3}{4}(2 m+4)=\frac{m+3}{2} m .
\end{aligned}
$$

$M(H)-m-(m+1)-(2 m+3)-(2 m+4)+1-c(H)=m>0$. Hence $H$ is not a complete intersection.

Case IV $m \equiv 0(\bmod 4)$. We put

$$
H=\left\langle m, m+1, n_{3}=\frac{(m-4)(m+1)}{2}+1, n_{4}=\frac{(m-2)(m+1)}{2}+2\right\rangle .
$$

Then $H$ is symmetric with;

$$
c(H)=m(m-3), \quad M(H)=h_{1}+h_{2}+h_{3},
$$

where

$$
\begin{aligned}
& h_{1}=m+n_{3}=(m+1) \frac{m-2}{2} \\
& h_{2}=m+n_{4}=2(m+1)+n_{3} \\
& h_{3}=c(H)+m=m(m-2)=n_{3}+n_{4} .
\end{aligned}
$$

$M(H)-m-(m+1)-n_{3}-n_{4}+1-c(H)=m>0$. Hence $H$ is not a complete intersection. 
THEOREM 2. For given positive integers $m$ and $n$, such that $m-$ $1 \geqq n \geqq 4$, there exists 1-dimensional local domain $R$ which is Gorenstein with $\operatorname{emb}(R)=n, m(R)=m$ and which is not a complete intersection.

Proof. It suffices to find a symmetric semigroup $H$ with $e m b(H)$ $=n$ and $m(H)=m$ and which is not a complete intersection.

(i) It is done for $n=4$ by Lemma 6 .

(ii) If $n \geqq m / 2$, this is true by Lemma 5 .

(iii) If $m / 2 \geqq n \geqq 4$, let $H_{1}=\langle n, n+1, \cdots, 2 n-2\rangle$. By Lemma 4, $H_{1}$ is symmetric with $c\left(H_{1}\right)=2 n$ and $e m b\left(H_{1}\right)=n-1$ which is not a complete intersection and $m \in H_{1}$. If we choose an integer $b$ so that $(b, m)=1$ and $b n\rangle m$, then $H=\left\langle m, b H_{1}\right\rangle$ is the desired example by Lemma 1.

Remark. The condition $m-1 \geqq n \geqq 4$ is necessary. If $n \geqq m$, we can choose $x \in R$ such that $m=m(R)=$ length $(R / x R)$. But as length $(R / x R) \geqq e m b(R)=n$, the only possibility is the case when $m=$ length $(R / x R)=e m b(R)$. But in this case the principal ideal $x R$ can not be irreducible and $R$ is not Gorenstein.

If $n=3$, then it is known by Serre that if $R$ is Gorenstein, then $R$ is a complete intersection.

\section{REFERENCES}

[1] J. Herzog and E. Kunz; Die Werthalbgruppe eines lokalen Rings der dimension 1, Sitzungsberichte der Heiderberger Akademie der Wissenschaften (1971), Springer-Verlag.

[2] J. Herzog; Generators and relations of abelian semigroups and semigrouprings. Manuscripta math. 3, 175-193 (1970).

[ 3 ] E. Kunz; The value-semigroup of a one-dimensional Gorenstein ring. Proc. A.M.S. 25, (1970) 748-751.

Tokyo Metropolitan University 